\section{El presente es cuántico}

Autora de la reseña: Ariadna Angulo-Brunet
Título completo de la obra reseñada:

Cuántica: tu futuro en juego

Autor: José Ignacio Latorre

Ciudad de publicación: Barcelona

Editorial: Editorial Planeta

Año: 2017

Número de páginas: 174

ISBN: 978-84-344-2539-2

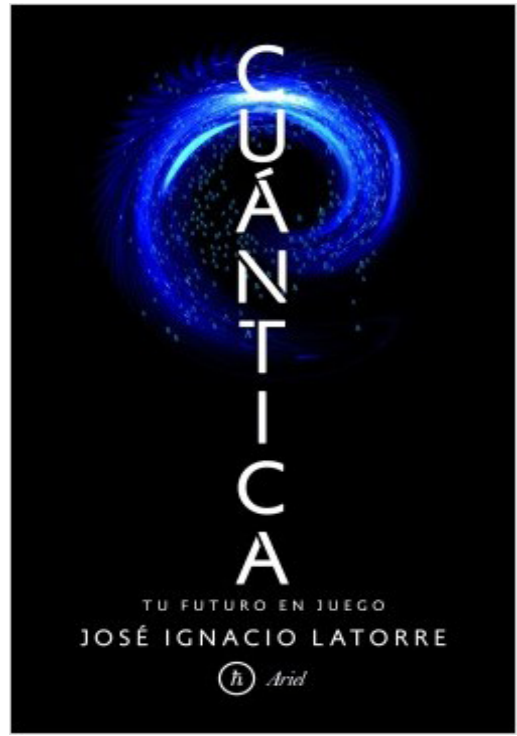

"El futuro será cuántico", eso afirma José Ignacio Latorre en la contraportada de Cuántica: tu futuro en juego, y eso logrará entender el lector que se adentre en esta obra de carácter divulgativo. Aunque la temática es compleja, el autor hace que los postulados de la mecánica cuántica sean un concepto relativamente sencillo para quienes no tienen conocimientos previos sobre la materia y permite valorar las implicaciones que tiene en el presente y en el futuro. Latorre es uno de los físicos españoles más reconocidos en la física cuántica. Su área de investigación se centra en las partículas elementales y en la teoría de la información cuántica. Actualmente está liderando un proyecto para desarrollar un ordenador cuántico, concretamente el procesador del ordenador. Mediante este libro se pone en evidencia la trayectoria de Latorre, quien da cuenta de que es posible divulgar conocimiento científico a cualquier público, referenciando teorías con un trasfondo matemático complejo, y sin necesidad de tener que comprender ni una sola operación aritmética.

El libro comienza con una pequeña introducción, donde el autor hace una analogía con el libro Las ciudades invisibles (Italo Calvino, 1972) y describe cómo podría haber sido una imaginaria ciudad de Cuántica. Como se ve a lo largo del texto, al describir la ciudad de Cuántica, Latorre nos ayudará a comprender las complejidades de la materia desde un relato más fantástico.

El prólogo del libro nos invita a reflexionar sobre la naturaleza cuántica de las cosas cotidianas; a darnos cuenta de que un teléfono móvil no podría existir sin la ciudad de Cuántica. Empieza desde el punto más elemental: saber el para qué y el porqué de la mecánica cuántica. $A$ continuación, siguen los cinco capítulos en que el autor estructura el contenido. El primero, y más largo, "Las leyes cuánticas", ilustra los cuatro postulados de la mecánica cuántica como si fueran las reglas elementales de la ciudad de Cuántica. El segundo, "La primera 
revolución cuántica" da a conocer la historia de la mecánica cuántica y nos hace entender sus aplicaciones prácticas actuales. En el tercero, "La segunda revolución cuántica" se discuten las implicaciones que puede tener en el futuro el uso de ordenadores cuánticos. En el cuarto "Mi futuro cuántico" el autor va más allá y describe en qué puede repercutir o cómo nos puede afectar la mecánica cuántica en el día a día. Finalmente, en el último capítulo, "La gran duda", el autor hace una reflexión de carácter general integrando los diferentes elementos que hemos ido conociendo sobre cuántica. Además de los agradecimientos, el libro también consta de dos apéndices: 1) Verdades cuánticas donde se hace hincapié en los postulados; y 2) Matemáticas cuánticas donde los más curiosos podrán ir más allá y conocer más sobre las matemáticas de la mecánica cuántica, por ejemplo los espacios de Hilbert.

A continuación vamos a empezar el viaje hacia la ciudad de Cuántica para ver de primera mano lo que, sobre ella, nos dice Latorre. El primer postulado de la mecánica cuántica nos indica el camino matemático a seguir. Establece qué podremos saber el comportamiento de un electrón, pero no sabremos lo que es ni por qué existe. Da cuenta de que la mecánica cuántica es un postulado humilde, "la mecánica cuántica no va a aportar las soluciones a nuestras preguntas existenciales" (p. 26). Para entender conceptos como las superposiciones cuánticas, entre otros, el autor revisa el clásico ejemplo del gato de Schrödinger:

Un gato es encerrado en una caja donde hay una botella con veneno -para ser precisos, Schrödinder escogió el ácido cian-hídrico-. En la caja hay un átomo radioactivo. Si este átomo decae y emite radiación, esa misma radiación dispara un contador Geiger. Este, a su vez dispara a un mecanismo con un martillo que cae sobre la botella, la rompe, esparce el veneno y mata el gato. Si el átomo no decae, todo sigue igual, y el gato deambula felizmente por la caja. (p. 32)

En este caso, y tal y como indica Latorre hay dos opciones posibles: que el gato esté vivo o que el gato esté muerto. No hay una contradicción, es una superposición. Aunque el primer postulado sea considerado el camino matemático, Latorre nos dice que: "Toda esta parafernalia de las matemáticas nos sirve para comprender, o al menos experimentar la ilusión de comprender" (p. 37). El segundo postulado aparentemente es más sencillo: "solo podemos obtener información de lo que realmente medimos" (p. 39). Por ejemplo, continúa el autor, no podemos saber qué ha pasado en una fiesta en la que no hemos estado. El tercer postulado determina que el azar existe y que el resultado de medir es aleatorio. Finalmente, en el cuarto postulado se denota que la evolución de la medición no tiene azar y que, por lo tanto, el tiempo es cambio. Como apunta Latorre: si hacemos dos fotografías y no observamos cambio es como si no hubiera pasado el tiempo.

"Los habitantes de la ciudad de Cuántica han ido aprendiendo a utilizar las leyes que han descubierto" (p. 65). Desde el día que nació la mecánica cuántica, el siete de octubre de 1900, cuando Max Planck estableció su constante, hasta hoy que utilizamos relojes atómicos situados en satélites para utilizar el GPS de nuestros móviles, hemos ido conociendo las extrañas leyes de la mecánica cuántica. Durante la primera mitad del siglo XX, científicos como Niels Bohr, Albert Einstein, Werner Karl Heisenberg o Erwin Schrödinger contribuyeron a lo que actualmente llamamos mecánica cuántica. El uso de láseres, resonancias magnéticas o la radioterapia han contribuido indudablemente a mejorar lo que llamamos medicina actual. Por otra parte, como el lector puede imaginarse, un campo en el que puede tener mucho peso la 
mecánica cuántica, son los ordenadores. Latorre nos dice que actualmente hay ordenadores que simulan comportamientos cuánticos y que son útiles para hacer operaciones aritméticas complejas.

Pero, Latorre se pregunta: ¿qué pasaría si tuviéramos un ordenador cuántico? A ello responde que la llamada segunda revolución científica se presenta como una "pacífica, práctica, no filosófica, pero de consecuencias difíciles de predecir" (p. 97). Un ordenador cuántico, aprovechando la superposición de elementos, nos permite hacer las operaciones muy rápido. Sin embargo, aunque esto tenga claras ventajas, son muchas las implicaciones negativas que puede tener si no se tiene en cuenta la seguridad de las comunicaciones. Aprovechando la capacidad de cálculo, con un ordenador cuántico podemos averiguar cualquier tipo de contraseña de un ordenador clásico:

Quien posea un ordenador cuántico podrá descifrar todas las transacciones secretas que se realizan por internet, $y$ las que se realizaron en el pasado y han sido almacenadas a la espera de un progreso disruptivo en el descifrado de la criptografía actual. (p. 100)

En este sentido, Latorre insiste en que es necesario avanzar en la búsqueda de ordenadores cuánticos, pero también de sistemas que nos permitan desarrollar una encriptación segura tanto para los ordenadores clásicos como para los cuánticos: una criptografía cuántica. El autor informa que, actualmente, ya existen algunos protocolos como pueden ser el BB84 o Ekert91.

Para terminar, el investigador discute cómo nos afectará en nuestra vida cotidiana la mecánica cuántica y, concretamente, los ordenadores cuánticos. Las preguntas que le pudieran haber ir quedando sueltas al lector del libro quedan, entonces, resueltas: ¿me afectará esto a mí? ¿Quedará alterada la política? ¿Habrá un negocio detrás? Y, lo más importante: ¿cuándo y quien lo hará? Aunque podemos debatir cómo será el futuro, llegados a este punto es evidente que mucha de la tecnología actual no existiría sin la mecánica cuántica. Latorre asegura que el futuro será cuántico, cuando en realidad el presente ya lo es.

Hay una creencia irracional a pensar que es difícil comprender la mecánica cuántica. Cuántica: tu futuro en juego, hace perder el miedo a conocer más sobre ella. No se puede pensar que visitar a Cuántica será sencillo, ya que la componen muchos elementos que se integran entre ellos, pero la sencillez con la que el autor describe los elementos hace que cualquier persona con interés científico pueda comprender el texto. Para el lector experto, Cuántica no le aportará nuevos conocimientos teóricos, pero podrá conocer la ciudad de Cuántica desde una perspectiva más crítica y amplia.

Como señala Latorre en el prólogo: "en el imaginario de las ciudades sutiles, Cuántica se fija de forma perenne en la memoria del viajero. Las disputas intelectuales de sus ágoras despiertan la imaginación, el afán de saber y la admiración" (p. 8).

Calvino, I. (2017). Las ciudades invisibles. Madrid: Siruela, XXIX edición. 
